\title{
Percepção da enfermagem da Atenção Básica referente a comunicação com paciente com transtorno mental: Revisão integrativa
}

\author{
Perception of Basic Care nursing regardling communication with mental disorders: Integrative
} review

Percepción de la enfermería de Cuidados Básicos sobre la comunicación con pacientes con transtornos mentales: Revisión integrativa

Recebido: 13/07/2021 | Revisado: 18/07/2021 | Aceito: 21/07/2021 | Publicado: 29/07/2021

\author{
Catarina Soares Araujo \\ ORCID: https://orcid.org/0000-0001-9205-7781 \\ Universidade Federal de Sergipe, Brasil \\ E-mail: catsoaraujo@gmail.com \\ Glebson Moura Silva \\ ORCID: https://orcid.org/0000-0002-4977-2787 \\ Universidade Federal de Sergipe, Brasil \\ E-mail: glebsonmoura@yahoo.com.br \\ Carla Kalline Alves Cartaxo Freitas \\ ORCID: https://orcid.org/0000-0001-7604-9132 \\ Universidade Federal de Sergipe, Brasil \\ E-mail: carlakalline@gmail.com \\ Daniella Silva Pereira \\ ORCID: https://orcid.org/0000-0002-6120-1207 \\ Universidade Federal de Sergipe, Brasil \\ E-mail:daniellapereiraenfufs@gmail.com
}

\begin{abstract}
Resumo
Objetivo: Identificar na literatura a percepção da equipe de enfermagem da Atenção Básica referente a comunicação com os pacientes com transtorno mentais. Métodos: Trata-se de uma revisão integrativa, realizada as buscas em meios eletrônicos, sendo eles: LILACS, CINHAL, SciELO e PubMed. Foram incluídos estudos do período de 2011 a 2021, nos idiomas: inglês, português e espanhol, que correspondessem a temática da percepção da equipe de enfermagem referente a comunicação com os pacientes com transtorno mental. E foram excluídos relatos de experiência e artigos duplicados. Resultados: A pesquisa resultou em 07 artigos que correspondem a temática da revisão, destes, foram encontrados nas bases de dados LILACS $(28,6 \%)$, CINHAL $(28,6 \%)$, SciELO $(28,6 \%)$ e PubMed $(14,2 \%)$. Sendo identificados 03 categorias para a análise de conteúdo: percepção da equipe de enfermagem quanto ao cuidado dos usuários com transtorno mental, relacionamento interpessoal entre profissionais de enfermagem e usuários com transtorno mental e comunicação como ferramenta importante para a realização do cuidado aos usuários com transtorno mental. Considerações finais: Observa-se que há poucos estudos que expõem sobre a percepção da equipe de enfermagem quanto a comunicação, e que existem barreiras para a realização do cuidado com pessoas com transtorno mental.
\end{abstract}

Palavras-chave: Enfermeiros da atenção básica; Pacientes com transtornos mentais; Comunicação.

\begin{abstract}
Objective: To identify in the literature the perception of the Primary Care nursing team regarding communication with patients with mental disorders. Methods: This is an integrative review, performed searches in electronic media, namely: LILACS, CINHAL, SciELO and PubMed. Studies from 2011 to 2021 were included, in the languages: English, Portuguese and Spanish, which corresponded to the theme of the nursing team's perception regarding communication with patients with mental disorders. And experience reports and duplicate articles were excluded. Results: The search resulted in 07 articles that correspond to the theme of the review, of which were found in the LILACS (28.6\%), CINHAL (28.6\%), SciELO (28.6\%) and PubMed databases (14,2\%). Three categories were identified for content analysis: perception of the nursing team regarding the care of users with mental disorders, interpersonal relationships between nursing professionals and users with mental disorders, and communication as an important tool for providing care to users with mental disorders. Final considerations: It is observed that there are few studies that expose the perception of the nursing team regarding communication, and that there are barriers to providing care for people with mental disorders. Keywords: Primary care nurses; Patients with mental disorders; Communication.
\end{abstract}




\begin{abstract}
Resumen
Objetivo: Identificar en la literatura la percepción del equipo de enfermería de Atención Primaria sobre la comunicación con pacientes con trastornos mentales. Métodos: Se trata de una revisión integradora, realizadas búsquedas en medios electrónicos, a saber: LILACS, CINHAL, SciELO y PubMed. Se incluyeron estudios de 2011 a 2021, en los idiomas: inglés, portugués y español, que correspondían al tema de la percepción del equipo de enfermería sobre la comunicación con pacientes con trastornos mentales. Y se excluyeron los informes de experiencia y los artículos duplicados. Resultados: La búsqueda resultó en 07 artículos que corresponden a la temática de la revisión, de los cuales se encontraron en las bases de datos LILACS $(28,6 \%)$, CINHAL $(28,6 \%)$, SciELO $(28,6 \%)$ y PubMed $(14,2 \%)$. Se identificaron tres categorías para el análisis de contenido: percepción del equipo de enfermería sobre la atención a los usuarios con trastornos mentales, relaciones interpersonales entre profesionales de enfermería y usuarios con trastornos mentales y la comunicación como herramienta importante para brindar atención a los usuarios con trastornos mentales. Consideraciones finales: Se observa que existen pocos estudios que expongan la percepción del equipo de enfermería sobre la comunicación, y que existen barreras para brindar atención a las personas con trastornos mentales.
\end{abstract}

Palabras clave: Enfermeras de atención primaria; Pacientes con trastornos mentales; Comunicación.

\title{
1. Introdução
}

Segundo a Portaria nº 2.436 (2017), a Atenção Primária à Saúde (APS) é caracterizada pelo conjunto de ações de saúde individuais, familiares e coletivas que abrangem a promoção, prevenção, proteção, diagnóstico, tratamento, reabilitação, redução de danos, cuidados paliativos e vigilância em saúde, de forma integral, a partir de uma gestão qualificada. Dessa forma, a APS torna-se a porta principal de entrada para ter acesso aos serviços do Sistema Único de Saúde (SUS), proporcionando saúde de forma integral e gratuita para todos os indivíduos.

Percebe-se que por ser a porta principal de entrada do SUS, a Atenção Básica (AB) é importante para os cuidados em saúde mental, pois gera diversas oportunidades para os profissionais estarem em contato com pessoas em sofrimento psíquico e vice-versa. Logo, o cuidado prestado na Atenção Básica referente a saúde mental, reflete uma possibilidade em modificação e qualificação nas condições e no modo de vida dos usuários (Ministério da Saúde, 2013).

Vale ressaltar que, segundo a Lei $\mathrm{n}^{\circ} 10.216$ (2001), as pessoas com transtorno mental devem ser reinseridas tanto na comunidade quanto em âmbito familiar e essa reinserção deve-se a reestruturação da assistência, de modo a garantir o cuidado de qualidade de forma integral e singular estabelecendo uma assistência que ultrapasse a lógica de internações de longa permanência, através da ordenação da Rede de Assistência à Saúde. Essa reestruturação da assistência em saúde mental dispõe sobre a garantia de cuidado de qualidade, de forma integral e singular, de forma a estabelecer uma assistência que ultrapasse a lógica de internações de longa permanência.

Os profissionais de enfermagem que atuam na Atenção Primária à Saúde devem tomar uma atitude positiva em relação à saúde mental. Essa atitude positiva do profissional é realizada desde como gestor através da elaboração de estratégias de educação permanente que promovam a mudança de atitudes dos profissionais da assistência na construção do cuidado a pessoa com transtorno mental de modo a permitir a inclusão, criação de vínculo, além de garantir o cumprimento dos direitos como cidadão portadores de transtorno, ou como atuante no cuidado direto. Dessa forma, produz uma inclusão, criação de vínculo, e garante que cumprimento dos direitos como cidadão portadores de transtorno ou não (Cassiano, Marcolan, \& Silva, 2019). Entretanto, uma grande porcentagem desses profissionais afirma não sentirem capacidade para atuar na saúde mental na Atenção Básica, devido à falta de aptidão para lidar com as questões relativas a saúde mental, e também a falta de capacitações voltadas ao atendimento qualificado que facilitem as ações de cuidado, o que gera barreiras que dificultam a ações de cuidados para essas pessoas (Nunes, Feitosa, Fernandes, Almeida, \& Ramos, 2020).

Uma forma do indivíduo manter contato em diversos tipos de situação é a comunicação, podendo ser verbal e não verbal. Logo, para que haja a construção de um vínculo entre o profissional de enfermagem com o usuário é preciso que haja a comunicação, o que torna a comunicação como um instrumento de cuidado. A enfermagem utiliza da comunicação na fala, postura corporal, expressão e até mesmo o silêncio. Entretanto, há barreiras que o profissional de enfermagem deve enfrentar 
que são: fatores psicológicos, linguagem, impedimento físico entre outros (Lima, 2013). Na saúde mental a comunicação também é um instrumento importante para a prestação da assistência e sem ela não há como estabelecer vínculo entre profissional e usuário (Stefanelli \& Carvalho, 2012).

Considerando os tópicos citados acima e a importância da comunicação para que haja uma assistência de qualidade para as pessoas com transtorno mental, almeja-se que haja mais pesquisas relacionadas à comunicação entre profissionais de enfermagem e pessoas com transtorno mental no âmbito da Atenção Primária à Saúde. Portanto, esta revisão tem como objetivo identificar a percepção dos profissionais de enfermagem acerca da comunicação com pacientes com transtorno mental e como essa comunicação pode afetar a assistência. Além disso, sintetizar os dados e evidências científicas sobre o tema.

\section{Metodologia}

Trata-se de uma revisão integrativa caracterizada por reunir estudos de diversas metodologias, ofertando aos pesquisadores a sintetização dos resultados com a finalidade de facilitar o aprofundamento do conhecimento. Além disso, tem o propósito de demonstrar lacunas em diversas áreas da pesquisa que necessitam de preenchimento. Para o desenvolvimento do estudo, foram realizadas as seguintes etapas: a identificação do tema, a criação da pergunta norteadora e dos critérios de inclusão e exclusão para a seleção da amostra, posteriormente, realizada a busca na literatura, classificação e avaliação dos estudos, análise dos resultados e a apresentação da síntese dos estudos (Mendes, Silveira, \& Galvão, 2008).

A pergunta norteadora utilizada foi: "qual a percepção dos profissionais de enfermagem da Atenção Básica quanto a comunicação com os pacientes com transtorno mental?". As buscas foram realizadas no período dos meses de janeiro de 2021 a março de 2021, em meios eletrônicos: (1) LILACS, (2) CINAHL, (3) PUBMED e (4) Scielo. Para a realização da busca, foram aplicados os descritores controlados pelos Descritores em Ciência da Saúde (DeCS) e os descritores controlados do Medical Subject Headings Section (MeSH): atenção primária à saúde, comunicação, saúde mental e equipe de enfermagem. E em relação ao operador aplicado, o AND foi utilizado.

A seleção primária dos estudos, foi realizada com a avaliação dos títulos e resumos, seguindo os critérios de inclusão/exclusão estabelecidos. Estes critérios são: artigos disponíveis em inglês, português e espanhol, publicados no período de 10 anos (2011-2021), foram excluídos estudos que fossem revisão sistemática, revisão integrativa e relato de caso, artigos duplicados e/ou não disponíveis na íntegra.

Para a realização da pesquisa, foi utilizado uma ferramenta das bases de dados (filtro), que auxilia na redução da quantidade de estudos que não correspondem ao objetivo da revisão. São eles: assunto principal, que permitia selecionar palavras que fizessem relação mais direta com o objetivo do estudo. As palavras utilizadas no filtro foram: comunicação, saúde mental, transtorno mental, atenção primária à saúde, relação interpessoal, relação profissional e paciente, atitude de profissionais de saúde, entre outros, resultando em um afunilamento mais objetivo no número de dados da pesquisa.

Em relação a seleção dos artigos, a estratégia PRISMA foi realizada, pois, serve como auxílio para que os autores melhorem o relato de revisões (Moher, Liberati, Tetzlaff, \& Altman, 2015). O fluxograma conforme o PRISMA é descrito informações sobre as etapas realizadas durante o processo da revisão, desde a identificação até a inclusão dos estudos na revisão, conforme a Figura 1 a seguir. 
Figura 1 - Fluxograma da estratégia PRISMA da seleção dos estudos para a inclusão na revisão.


Fonte: Autores (2021).

Os estudos selecionados para a inclusão na revisão, foram organizados em um quadro contendo nome do autor, ano de publicação, método, objetivo, resultados, nível de evidência e categorização sobre a percepção dos profissionais de enfermagem quanto a comunicação com pacientes com transtorno mental na Atenção Básica.

A revisão integrativa precisa ser realizada de forma ordenada, com isso, considera-se as características de cada estudo com rigor. Para que fosse realizado um sistema de classificação de evidências, foram identificados de modo hierárquico, conforme a abordagem metodológica utilizada. Logo, para que haja a melhor escolha possível de estudo com mais evidência, sugere-se uma organização, conforme o delineamento da pesquisa. A classificação de evidências possui cinco níveis: nível 1 para estudos de meta-análises; nível 2 para estudos individuais com delineamento experimental; nível 3 para estudos quaseexperimentais; nível 4 para estudos descritivos ou com abordagem qualitativa; nível 5 para estudos baseados em opiniões de especialistas (Stetler et al, 1998).

$\mathrm{Na}$ busca de uma interpretação das informações, foram adotados eixos transversais na análise das diferentes áreas temáticas envolvidas neste estudo, com categorização das informações, propostas no método de análise de conteúdo de Bardin (2011) quando nessa perspectiva busca descrever e sistematizar o conteúdo manifesto das comunicações, tendo por finalidade a interpretação das mesmas. A autora sugere as seguintes fases para tratamento das informações: Fase Pré-análise: leitura das respostas encontradas nos estudos selecionados, organizando o material de forma criteriosa, procurando semelhança entre as ideias expressadas; Fase de Exploração de material: o momento da codificação, na qual ocorreu a transformação dos dados coletados em conteúdos sistemáticos. Os dados foram agrupados em temáticas, intitulados por categorias que expressaram a essência dos conteúdos das respostas; Fase do tratamento dos resultados, inferência e interpretação: diante dos resultados adquiridos ocorreram as interpretações, baseadas na fundamentação teórica que norteou a pesquisa. 


\section{Resultados}

Com a realização da pesquisa, foram identificados um total de 29.567 estudos, destes foram excluídos 442 estudos por duplicidade. Além disso, foram excluídos também 17.213 artigos durante a triagem, devido a utilização dos filtros supracitados. Visto que as combinações dos descritores deixaram o resultado muito amplo, abrindo espaço para assuntos que não correspondiam a pesquisa.

Com isso, resultou em um total de 11.207 artigos para a leitura de títulos e resumos. Os estudos foram excluídos por não responderem à pergunta norteadora, pois, a grande maioria dos estudos abordava a comunicação entre setores, comunicação entre profissionais, e quando abordava a comunicação com paciente com transtorno, não envolvia a Atenção Primária, e sim a área intra e extra hospitalar. Resultando, para a realização da leitura do artigo na íntegra, 16 estudos que contemplavam todos os critérios de exclusão/inclusão. Os estudos identificados em sua maioria, com 72,1\% do total, na base de dados CINHAL. O restante dos estudos identificados expressa 27,9\% do total, sendo 21,5\% da Pubmed, 5,5\% da LILACS e 0,9\% da SciELO.

Em relação a leitura na íntegra dos estudos, foram selecionados 16 artigos. Destes, cerca de 56,25\% são artigos em português, 37,5\% em inglês e apenas 6,25\% encontra-se com idioma em espanhol. Em relação aos métodos identificados nos estudos, em sua grande maioria, são estudos de caráter qualitativo, em um total de $93,75 \%$, e o restante dos estudos, com $6,25 \%$ são estudos quantitativos.

Em relação ao nível de evidência, cerca de $75 \%$ estão no nível 4, com 18,75\% estão no nível 5, e apenas 6,25\% identifica-se como nível 1. Além disso, foi identificado com essa pesquisa observa-se que o Brasil contempla grande parte dos estudos que envolvem a percepção da equipe de enfermagem quanto a comunicação com paciente com transtorno mental, com cerca de $75 \%$, e o restante dos países que obtém estudos sobre esse tema foram os Estados Unidos da América, Suécia e Inglaterra com $12,5 \%, 6,25 \%, 6,25 \%$ respectivamente.

Os artigos incluídos na revisão foram 07 estudos, pois contemplaram de forma efetiva os critérios de exclusão/inclusão. Sendo assim, estes artigos são todos identificados com abordagem qualitativa, com o nível 4 de evidência. Além disso, com cerca de $85,7 \%$ dos estudos são escritos utilizando o idioma português e com menos de 15\%, o idioma identificado é o inglês. Os estudos incluídos nesta revisão foram organizados a partir do delineamento do estudo para facilitação do entendimento, conforme o Quadro 1.

Quadro 1 - Artigos utilizados na revisão integrativa.

\begin{tabular}{|c|c|c|c|c|c|c|}
\hline Ano & Autores & Método & Objetivo & Resultados & $\begin{array}{c}\text { Nível de } \\
\text { evidência }\end{array}$ & Categorização \\
\hline 2014 & $\begin{array}{l}\text { VILELA; } \\
\text { PEDRÃO; } \\
\text { CARVALHO }\end{array}$ & $\begin{array}{l}\text { Estudo } \\
\text { descritivo, de } \\
\text { abordagem } \\
\text { qualitativa. }\end{array}$ & $\begin{array}{l}\text { Compreender a percepção } \\
\text { dos enfermeiros da } \\
\text { Estratégia da Saúde da } \\
\text { Família sobre o cuidado à } \\
\text { pessoa com diagnóstico de } \\
\text { transtorno mental. }\end{array}$ & $\begin{array}{l}\text { Observou-se que as enfermeiras } \\
\text { sinalizam sobre a dificuldades em } \\
\text { relação a conhecimentos } \\
\text { específicos de saúde mental, bem } \\
\text { como sentimentos de medo, } \\
\text { impotência. }\end{array}$ & Nível 4 & $\begin{array}{l}\text { Conhecimentos } \\
\text { sobre saúde mental } \\
\qquad \mathrm{X} \\
\text { Sentimento de } \\
\text { medo e impotência }\end{array}$ \\
\hline 2017 & $\begin{array}{l}\text { JANLÖV; } \\
\text { JOHANSSON; } \\
\text { CLAUSSON }\end{array}$ & $\begin{array}{l}\text { Abordagem } \\
\text { exploratória } \\
\text { qualitativa }\end{array}$ & $\begin{array}{l}\text { Explorar o distrito a } \\
\text { experiência das enfermeiras } \\
\text { de encontrar e lidar com } \\
\text { doença mental entre } \\
\text { pacientes adultos em } \\
\text { cuidados de saúde centros. }\end{array}$ & $\begin{array}{l}\text { As enfermeiras expõem a } \\
\text { necessidade de ter competência, } \\
\text { tempo compromisso além de um } \\
\text { lugar organizado e preparado. } \\
\text { Além dos sentimentos de medo e } \\
\text { incerteza sobre como realizar a } \\
\text { comunicação com os pacientes } \\
\text { com transtorno mental. }\end{array}$ & Nível 4 & $\begin{array}{c}\text { Competência para } \\
\text { o trabalho em } \\
\text { saúde mental } \\
\text { X } \\
\text { Sentimento de } \\
\text { medo e impotência }\end{array}$ \\
\hline
\end{tabular}




\begin{tabular}{|c|c|c|c|c|c|c|}
\hline 2019 & $\begin{array}{l}\text { CASSIANO; } \\
\text { MARCOLAN; } \\
\text { SILVA }\end{array}$ & $\begin{array}{l}\text { Estudo } \\
\text { qualitativo, } \\
\text { do tipo } \\
\text { reflexivo }\end{array}$ & $\begin{array}{l}\text { Refletir a relação entre } \\
\text { pacientes portadores de } \\
\text { transtornos mentais e } \\
\text { enfermeiros, r pelo } \\
\text { atendimento nas unidades } \\
\text { de Atenção Primária à } \\
\text { Saúde. }\end{array}$ & $\begin{array}{l}\text { Observa-se que a equipe de } \\
\text { enfermagem possui estigmas e } \\
\text { despreparo em relação ao cuidado } \\
\text { aos pacientes com transtorno } \\
\text { mental. Dificultando a } \\
\text { comunicação interpessoal e } \\
\text { cuidado de qualidade. }\end{array}$ & Nível 4 & $\begin{array}{c}\text { O estigma como } \\
\text { empecilho na } \\
\text { comunicação }\end{array}$ \\
\hline 2016 & SILVA et al & $\begin{array}{l}\text { Estudo } \\
\text { qualitativo }\end{array}$ & $\begin{array}{l}\text { Conhecer a percepção dos } \\
\text { profissionais de saúde da } \\
\text { família acerca da } \\
\text { implementação de ações de } \\
\text { saúde mental na Atenção } \\
\text { Primária à Saúde. }\end{array}$ & $\begin{array}{l}\text { A equipe de enfermagem relata } \\
\text { dificuldades para lidar com o } \\
\text { paciente com transtorno mental, } \\
\text { devido a capacitação insuficiente, } \\
\text { além dos sentimentos de medo, } \\
\text { angústia. }\end{array}$ & Nível 4 & $\begin{array}{l}\text { Competência para } \\
\text { o trabalho em } \\
\text { saúde mental } \\
\text { X } \\
\text { Sentimento de } \\
\text { medo e impotência }\end{array}$ \\
\hline 2021 & $\begin{array}{l}\text { BOSSATO; } \\
\text { OLIVEIRA; } \\
\text { DUTRA; } \\
\text { LOYOLA. }\end{array}$ & $\begin{array}{l}\text { Estudo } \\
\text { qualitativo }\end{array}$ & $\begin{array}{l}\text { Analisar as ações da equipe } \\
\text { de Enfermagem que } \\
\text { promovem o protagonismo } \\
\text { do usuário no Centro de } \\
\text { Reabilitação Psicossocial. }\end{array}$ & $\begin{array}{l}\text { A comunicação criativa, o } \\
\text { trabalho em rede e a esquipe de } \\
\text { enfermagem como antena, são } \\
\text { necessários para que o paciente } \\
\text { com transtorno mental consiga } \\
\text { autonomia e para que haja um } \\
\text { cuidado de qualidade. }\end{array}$ & Nível 4 & $\begin{array}{l}\text { Comunicação } \\
\text { criativa e } \\
\text { autonomia do } \\
\text { usuário }\end{array}$ \\
\hline 2020 & $\begin{array}{l}\text { PEREIRA, } \\
\text { AMORIM, } \\
\text { GONDIM }\end{array}$ & $\begin{array}{l}\text { Estudo } \\
\text { qualitativo }\end{array}$ & $\begin{array}{l}\text { Conhecer a prática dos } \\
\text { profissionais sobre Saúde } \\
\text { Mental na Atenção Primária } \\
\text { à Saúde contribuindo para o } \\
\text { esclarecimento das } \\
\text { dificuldades em suas rotinas } \\
\text { e para melhorar a } \\
\text { assistência aos usuários. }\end{array}$ & $\begin{array}{l}\text { A equipe de enfermagem sabe a } \\
\text { necessidade da atuação em saúde } \\
\text { mental. Entretanto, alegam possuir } \\
\text { capacitação defasada, medo de } \\
\text { contato com os usuários e não ter } \\
\text { conhecimento sobre a Rede de } \\
\text { Atenção Psicossocial. }\end{array}$ & Nível 4 & $\begin{array}{l}\text { Competência para } \\
\text { o trabalho em } \\
\text { saúde mental } \\
\text { X } \\
\text { Sentimento de } \\
\text { medo e impotência }\end{array}$ \\
\hline 2020 & NUNES et al & $\begin{array}{l}\text { Estudo } \\
\text { qualitativo }\end{array}$ & $\begin{array}{l}\begin{array}{l}\text { Descrever e } \\
\text { atuação do enfermeiro }\end{array} \\
\text { especialista em saúde } \\
\text { mental a Estratégia Saúde } \\
\text { da Família. }\end{array}$ & $\begin{array}{l}\text { Os enfermeiros possuem conceitos } \\
\text { psiquiátricos embasados no } \\
\text { modelo biológico. Além de } \\
\text { relatarem a falta de capacitação } \\
\text { em saúde mental e os estigmas } \\
\text { para lidar com os pacientes com } \\
\text { transtorno mental. }\end{array}$ & Nível 4 & $\begin{array}{l}\text { O estigma como } \\
\text { empecilho na } \\
\text { comunicação }\end{array}$ \\
\hline
\end{tabular}

Fonte: Autores (2021).

Para a realização da análise do conteúdo dos 07 estudos, foram separados por categorias: categoria 1 para a percepção da equipe de enfermagem quanto ao cuidado dos usuários com transtorno mental; categoria 2 para o relacionamento interpessoal entre profissionais de enfermagem e usuários com transtorno mental; categoria 3 para a comunicação como ferramenta importante para a realização do cuidado aos usuários com transtorno mental. Dessa forma, obteve-se, cerca de 57,2\% dos estudos identificados como categoria 1 , em relação a categoria 2 , observa-se cerca de $28,5 \%$ e o restante dos estudos encaixam-se na categoria 3.

\section{Discussão}

Com a realização da análise de conteúdo foi possível observar que a maioria dos estudos que contemplam os objetivos, estão na categoria 1, ou seja, são estudos que evidenciam sobre a percepção da equipe de enfermagem quanto ao cuidado com os usuários com transtorno mental. Como por exemplo, Nunes et al (2020) afirma em seu estudo que a equipe de enfermagem refere resistência para prestar o cuidado aos usuários em sofrimento psíquico. Apesar dos profissionais terem conhecimento 
sobre a importância do cuidado em saúde mental, além de terem ciência que os profissionais da Atenção Básica possuem maior aproximação com toda a população. Logo, como a Atenção Primária à Saúde se dispõe como uma porta de entrada para os serviços de saúde. E ela possui uma demanda crescente de quase $20 \%$ dos usuários que necessitam de cuidado em saúde metal. Sendo assim, é importante que os profissionais da Atenção Primária estejam preparados para lidar com as necessidades desse público (Ministério da Saúde, 2013).

Quando ainda se trata sobre a percepção dos profissionais de enfermagem, Pereira, Amorim e Gondim (2020), referem em seu estudo que os enfermeiros relatam possuir dificuldade na comunicação, criação de vínculos, e não se sentem aptos para atuar na saúde mental. Além disso, observa-se que quase $90 \%$ dos autores da revisão integrativa evidenciam em suas pesquisas que as equipes de enfermagem têm dificuldades na área de saúde mental. Entretanto, para que haja a consolidação do direito universal à saúde, os profissionais devem ter conhecimento e habilidades, garantindo à população um cuidado integral (Lei n. 10.2016, 2001).

Os profissionais de enfermagem, em sua grande maioria, não se sentem aptos para atuar na saúde mental, por diversos fatores, entre eles a falta de preparo, desde capacitações, treinamentos e atualizações. E essa falta de preparo pode ser causada pela falta de apoio da gestão ou até mesmo da ausência na formação acadêmica, ou fluxo pequeno de indivíduos que utilizam da rede de saúde (Nunes et al, 2020).

Vale ressaltar que profissionais de enfermagem referiam, em quase todos os estudos, que têm dificuldades na comunicação com os usuários com transtorno mental. Mas, os entrevistados deixaram explícitos que a comunicação é importante para o cuidado humanizado e de qualidade. No estudo de Vilela, Pedrão e Carvalho (2014), evidencia-se de forma clara que os enfermeiros entendem que a comunicação, o acolhimento, a escuta livre e o uso de tecnologias leves são essenciais para o cuidado de pessoas com transtorno mental. Sendo assim, os profissionais da APS necessitam desenvolver competências para poder lidar com pessoas em sofrimento psíquico, além de deixar-se receber auxílio de outros profissionais mais experientes quando precisar, garantindo um cuidado integralizado (Macinko, \& Mendonça, 2018).

Silva, Reis, Santos, Souza, e Azevedo (2016), expõe em seu estudo que os enfermeiros possuem grande dificuldade na relação interpessoal, pois, não possuem habilidades na comunicação com pessoas em sofrimento psíquico. Os enfermeiros neste estudo referem receio de comunicar-se e até mesmo realizar um simples toque no paciente. Essa falta de habilidade afeta o cuidado, desde o acolhimento até a criação de vínculo. Logo, observa-se que o cuidado humanizado em saúde mental e educação permanente em saúde mental é necessário para que haja um cuidado de qualidade. (Lucigan, Toledo, \& Garcia, 2012; Luz \& Miranda, 2010).

Outra categoria definida para análise de conteúdo é a categoria 2, com 28,5\% dos estudos, o qual se refere ao relacionamento interpessoal entre profissional de enfermagem e usuário com transtorno mental. Por exemplo, no estudo de Cassiano et al, (2019) constata-se que os enfermeiros entrevistados relatam sobre a existência de um distanciamento com os pacientes com transtorno mental, devido a algumas barreiras. As barreiras citadas pelos enfermeiros foram sobre a formação acadêmica ineficaz, uma concepção social discriminatória, e a falta de capacitação dos profissionais, ou seja, falta de educação permanente nas instituições. Essas barreiras podem ser reflexo de uma sociedade que define a pessoa com transtorno mental, alguém que põe medo, que dá vergonha, que não tem direitos, que não tem prognóstico, ou até mesmo sem identidade, ocasionando uma descrença no cuidado e no interesse a estas pessoas (Paiva et al, 2016).

Os pacientes que estão em sofrimento psíquico instigam um olhar com diversos estigmas e preconceitos que acabam tornando-se barreiras do cuidado. Sendo assim, observa-se a necessidade de capacitação para os profissionais que possibilite a realização do cuidado com os usuários em sofrimento que possam lidar com as angústias e tornar o profissional mais confiante de seus conhecimentos e habilidades (Matuda, Pinto, Martins, \& Frazão, 2015). Para que isso ocorra, o uso das tecnologias leves na Atenção Primária à Saúde pode garantir um cuidado com mais qualidade (Pereira et al,2020) 
As atribuições do enfermeiro da Atenção Básica podem ser divididas em duas categorias, a produção do cuidado e gestão terapêutico, e as atividades de gerenciamento do serviço de saúde e equipe de enfermagem (Ferreira, Perico, \& Dias, 2018). Quando relacionado com a Saúde Mental não é diferente, o enfermeiro deve garantir um cuidado continuado de qualidade para as pessoas em sofrimento psíquico. Sendo assim, com menos de $15 \%$ dos estudos, a categoria 3 , mostra sobre a comunicação como ferramenta importante para o cuidado do usuário com transtorno mental. E essa afirmação pode ser confirmada no estudo de Bossato, Oliveira, Dutra e Loyola (2021), o qual eles mostram os enfermeiros que utilizam a comunicação criativa para manter um cuidado de qualidade às pessoas com transtorno mental. Além disso, esse estudo mostra a importância da comunicação e as formas que o enfermeiro pode utilizá-las, como uma comunicação não verbal, a utilização de desenhos, para que enfim possa tornar o paciente com transtorno mental protagonista do seu próprio tratamento.

Logo, é necessário que a equipe de enfermagem realize uma mudança de atitude, procure melhorar as habilidades e competências em saúde mental (Cassiano et al, 2019). Ou iniciar a mudança com um simples questionamento sobre sua real competência de comunicação e o quanto ela pode afetar a qualidade da assistência. Como no estudo de Jonlov e Clausson (2017), o qual expõe a preocupação de enfermeiros em relação a comunicação eficaz com pacientes com transtorno mental, e até onde essa comunicação pode garantir um retorno ao serviço, tornando um cuidado continuado e de qualidade. Além de afirmarem a necessidade que todos os profissionais que atuam com a saúde mental sejam capacitados sobre a comunicação verbal e não verbal.

\section{Considerações Finais}

Com essa pesquisa pode-se observar que são poucos os estudos que expõem sobre a percepção da equipe de enfermagem quanto a comunicação com pacientes com transtorno mental. Sendo assim, os estudos selecionados para a revisão evidenciam que os profissionais de enfermagem encontram diariamente na Atenção Primária a Saúde barreiras para a realização do cuidado com pessoas em sofrimento psíquico. As barreiras que as equipes de enfermagem têm que lidar quando se refere com problemas na comunicação com os pacientes em sofrimento psíquico, são barreiras como medo, falta de confiança, falta de capacitações, estigmas e preconceitos, entre outros. Logo, esses problemas acabam afetando a criação de vínculo entre pacientes e profissionais de enfermagem, podendo desencadear um cuidado defasado às pessoas com transtorno mental.

Entretanto, é possível também observar nesse estudo que os profissionais de enfermagem têm ciência sobre a importância da comunicação para os cuidados com as pessoas em sofrimento psíquico. Dessa forma, eles expõem que necessitam de capacitações referentes a saúde mental e capacitações de comunicação verbal e não verbal. Além de expor a importância do uso da comunicação criativa para que haja um cuidado eficaz.

Com essa perspectiva, reflexões são feitas sobre esse tema pouco abordado pelos estudiosos. A comunicação, por ser uma habilidade inata do ser humano, não necessita de estudos? Os profissionais não precisam da comunicação para o cuidado? E quando envolve a saúde mental, a comunicação não é uma ferramenta importante para a criação de vínculos e, consequentemente, para um cuidado continuado e integral? Logo, é necessário que haja mais pesquisas envolvendo a comunicação entre a equipe de enfermagem e as pessoas com transtorno mental para que o cuidado na Atenção Básica seja embasado cientificamente, tornando um cuidado de qualidade, humanizado, contínuo e integral.

\section{Referências}

Bardin, L. (2011). Análise de Conteúdo. 70, 229.

Bossato, H. R., Oliveira, R. M. P., Dutra, V. F. D., \& Loyola, C. M. D. (2021). A enfermagem e o protagonismo do usuário no CAPS: um estudo na perspectiva construcionista. Rev Gaúcha Enferm. 42. 
Cassiano, A. P. C., Marcolan, J. F., \& Silva, D. A. (2019). Atenção primária à saúde: estigma a indivíduos cm transtornos mentais. Revista de Enfermagem Ufpe. 13(1), 1-6.

Ferreira, S. R. S., Perico, L. A. D., \& Dias, V. R. F. G. (2018). The complexity of the work of nurses in Primary Health Care. Rev. Bras. Enferm. 71(1), 74-709.

Jonlov, A. C., Clausson, L. J. K. (2017). Mental health problems among adult patients in Sweden: experiences of district nurses. Scand J Caring Sci. 32, 987996.

Lei n. 10.2016, de 06 de abril de 2001 (2001). Dispõe sobre a proteção e os direitos das pessoas portadoras de tarsntornos mentais e redireciona o modelo assistencial em saúde mental. Brasília.

Lima, P. M. S. (2021). Fatores que interferem na comunicação do enfermeiro com o paciente de terapia intensiva. Trabalho de conclusão de curso (TCC) na pós-graduação em Enfermagem. Pós-graduação Lato Sensu.

Lucigan, D. H. I., Toledo, V. P., \& Garcia, A. P. R. F. (2021). Acolhimento e saúde mental: desafio profissional na Estratégia Saúde da Família. Rev Rene. 13(1), $02-10$.

Luz, P. M., \& Miranda, K. C. L. (2010). As bases filosóficas e históricas do cuidado e a convocação de parceiros sexuais em HIV/aids como forma de cuidar. Ciênc. saúde coletiva. 15(1). 1143-1148.

Macinko, J., Mendonça, C. S. (2018). Estratégia Saúde da Família, um forte modelo de Atenção Primária à Saúde que traz resultados. Saúde debate. 42, 18-37.

Matuda, C. G., Pinto, N. R. S., Martins, C. L., \& Frazão, P. (2015). Colaboração interprofissional na Estratégia Saúde da Família: implicações para a produção do cuidado e a gestão do trabalho. Ciênc, saúde coletiva. 20(8). 2511-2521.

Mendes, K. D. S., Silveira, R. C. C. P., \& Galvão, C. M. (2008). Revisão integrativa: método de pesquisa para a incorporação de evidências para a incorporação de evidências na saúde e na enfermagem. Texto \& Contexto - Enfermagem. 17(4), 758-764.

Ministério da Saúde (2013). Saúde Mental. Caderno de Atenção Básica. Secretaria de Atenção à Saúde, Departamento de Atenção Básica. 2013.

Moher, D., Liberati, A., Tetzlaff, J., \& Altman, D. G. (2015). Principais itens para relatar Revisões sistemáticas e Meta-análises: a recomendação prisma. Epidemiologia e Serviços de Saúde. 24(2), 335-342.

Nunes, V. V., Feitosa, L. G. G. C., Fernandes, M. A., Almeida, C. A. P. L., \& Ramos, C. V. (2020). Saúde mental na atenção básica: atuação do enfermeiro na rede de atenção psicossocial. Rev. Brasileira de Enfermagem. 73, 1-7.

Paiva, P. C., Torrenté, M. O. N, Landim, F. L. P. Branco, J. G. O., Tambori, B. C. R., \& Cabral, A. L. T. (2016). Sofrimento psíquico e a abordagem da comunidade na voz do agente comunitário de saúde. Rev Esc Enferm Usp. 50, 139-144.

Pereira, R. M., Amorim, F. F., \& Gondim, M. F. N. (2020). A percepção e a prática dos profissionais da Atenção Primária sobre a Saúde Mental. Interface. 4, $1-13$.

Portaria n. 2.436, de 21 de setembro de 2017 (2017). Aprova a Política Nacional de Atenção Básica, estabelecendo a revisão de diretrizes para a organização da Atenção Básica, no âmbito do Sistema Único de Saúde (SUS). Brasília. 2017.

Silva, G. R., Reis, H. F. T., Santos, E. M., Souza, M. P. A., \& Azevedo, R. L. (2016). Saúde Mental na Atenção Primária: percepções da equipe de Saúde da Família. Cogitare Enferm. 21(2), 01-07.

Stefanelli, M. C.; Carvalho, E. C. (2012). A contribuição nos diferentes contextos da enfermagem. Barueri: Manole. 2.

Stetler, C. B., Morsi, S. Rucki, S., Broughton, S., Corrigan, B, Fitzgerald, J., Giuliano, K., Havener, P., \& Sheridan, A. (1998). Utilization-focused integrative reviews in a nursing service. Applied Nursing Research. 11(4), 195-206.

Vilela, S. C., Pedrão, L. J., \& Carvalho, A M. P. (2014). O cuidado da enfermagem em saúde mental na atenção básica: conceitos, vivência e práticas. Rev de Enfermagem Ufpe. 